\title{
ON THE BARRIER TO EXCITED-STATE INTRAMOLECULAR PROTON TRANSFER (ESIPT): THE CONTROVERSIAL ASSIGNMENT OF THE SPECTRA OF 2,5-BIS(2-BENZOXAZOLYL)HYDROQUINONE IN SOLID ARGON
}

\author{
Bernhard DICK \\ Max-Planck-Institut für Biophysikalische Chemie, Abteilung Laserphysik, D-3400 Göttingen, Federal Repuhlic of Germany
}

Received 2 March 1989

\begin{abstract}
The spectra of 2,5-bis (2-benzoxazolyl)hydroquinone (BBXHQ) in solid argon at $15 \mathrm{~K}$ reveals two species. The major species $(96 \%)$ undergoes very efficient excited-state intramolecular proton transfer (ESIPT) after excitation to $S_{1}$. It is assigned to the isolated BBXHQ molecule (solvated by argon atoms) in which ESIPT is not thermally activated. Either no energy barrier exists or it can be overcome by proton tunneling with a rate constant greater than $8 \times 10^{10} \mathrm{~s}^{-1}$. The minor species produces only fluorescence with a normal Stokes shift, indicating the absence of ESIPT. It is tentatively assigned to a dimer of BBXHQ.
\end{abstract}

\section{Introduction}

The excited-state intramolecular proton transfer (ESIPT) reaction in the molecule 2,5-bis(2-benzoxazolyl)hydroquinone (BBXHQ) has drawn considerable attention in recent years [1-8]. After excitation of the molecule in its normal form $\mathrm{X}$ to the lowest excited singlet state ${ }^{1} \mathrm{X}^{*}$, the proton migrates along the hydrogen bond to form the tautomer in the lowest excited singlet state ' $\mathrm{Y}$ '. In all other molecules that show this effect the ESIPT reaction ${ }^{1} \mathrm{X}^{*} \rightarrow{ }^{1} \mathrm{Y}^{*}$ is very fast cven at $15 \mathrm{~K}\left(k_{\mathrm{XY}} \approx 10^{12} \mathrm{~s}^{-1}\right)$ except for molecules with identical structures for $\mathrm{X}$ and $Y[9-11]$. As a consequence no fluorescence $F_{X}$ from ${ }^{~} \mathrm{X}^{*}$ is observed, but only strongly Stokes-shifted fluorescence $F_{Y}$ from the excited tautomer ${ }^{1} Y^{*}$. BBXHQ and its monomethoxy derivative are the only exceptions reported so far: In the temperature range 143-293 $\mathrm{K}$ two fluorescences, $F_{\text {blue }}$ and $F_{\text {red, }}$, with equal decay times and equal excitation spectra were observed $[1,2]$. Equilibrium between the two fluorescing species is established within a few picoseconds. The blue fluorescence was assigned to $F_{X}$ and the red fluorescence to $F_{Y}$. From the temperature dependence of the quantum yield of blue fluorescence a reaction enthalpy for ESIPT of $\Delta H=-0.5$ $\mathrm{kcal} / \mathrm{mol}$ was deduced [3]. The height of the postulated barrier could not be obtained in this way.
A convincing proof for the existence of a barrier to ESIPT should be the observation of an increasing fluorescence quantum yield ratio $\Phi_{\mathrm{X}} / \Phi_{\mathrm{Y}}$ with decreasing temperature. The opposite behavior is observed for BBXHQ in the temperature range 143-293 $\mathrm{K}$, since the reaction is exothermic and equilibrium between both forms is established very rapidly. At sufficiently low temperatures, however, the back-reaction should no longer occur.

Low-temperature experiments must carefully avoid the formation of complexes of BBXHQ with hydrogen-bonding impurities. Matrix isolation in solid argon is a simple and efficient method for this purpose as has been demonstrated with 3-hydroxyflavone $[12,13]$ which is especially sensitive to complex formation [14]. Excitation spectra of BBXHQ in solid argon revealed the existence of $t$ wo ground-state species [5]: Species A $(\approx 96 \%)$ yields only red fluorescence from the tautomer form; hence ESIPT is a very rapid process in these molecules even at $15 \mathrm{~K}$. Species $B(\approx \mathbf{4} \%)$ emits only blue fluorescence which is mirror symmetric to its excitation spectrum; hence ESIPT is inhibited in these molecules. Species $A$ was assigned to isolated $\mathrm{BBXHQ}$, whereas species $\mathrm{B}$ was tentatively assigned to a dimer or conformer incapable of undergoing ESIPT [5].

This assignment has been criticized in a recent article by Grabowska, Mordzinski, Tamai and Yoshi- 
hara (GMTY) [6]. They assigned the major species to "perturbed" BBXHQ-argon 1: 1 complexes and the minor species to unperturbed "isolated" BBXHQ. The present article has the following objectives:

(i) To show that the work of GMTY [6] provides no evidence against the original interpretation [5] of the spectra of BBXHQ isolated in solid argon.

(ii) The reinterpretation is inconsistent with the experimental data.

(iii) The interpretation in ref. [5] is not in contradiction with the model of reversible ESIPT at high temperatures, as supposed by GMTY.

(iv) An analysis of the published data on the temperature dependence of the fluorescence quantum yields suggests that the simple model of reversible ESIPT in BBXHQ is incomplete.

\section{The spectra of BBXHQ isolated in solid argon}

The sample preparation and experimental procedure applied to the study of BBXHQ isolated in solid argon have been described [5]. The results are presented here in greater detail to provide an undisputable basis for interpretation.

Argon matrices doped with BBXHQ emit bright red fluorescence when exposed to IV V light. The excitation spectrum (ES) of this red fluorescence, detected at $585 \mathrm{~nm}$, is shown as ES(a) in fig. 1. Two electronic transitions can be distinguished, the first with broad and diffuse vibrational structure between 23000 and $29000 \mathrm{~cm}^{-1}$, the second with several distinct vibronic lines between 29000 and $35000 \mathrm{~cm}^{-1}$. The strongest feature is a sharp line at $331.9 \mathrm{~nm}$ belonging to the second transition. The corrected fluorescence spectrum (FS) obtained with excitation into this strong line is shown as $\mathrm{FS}$ (c). It consists of a strong band with a peak at $580 \mathrm{~nm}$ corresponding to a Stokes shift of $\approx 7000 \mathrm{~cm}^{-1}$. Hence ES (a) belongs to a species (called species $A$ ) which undergoes a very efficient ESIPT reaction even at $15 \mathrm{~K}$. FS (c) shows also a weak emission in the spectral region between 19000 and $24000 \mathrm{~cm}^{-1}$, where the fluorescence of the normal form of $B B X H Q$ is expected. ES(b) is the excitation spectrum of the blue fluorescence, detected at $460 \mathrm{~nm}$. Its appearance is very similar to that of ES (a) but the band positions are significantly red-shifted. The strongest feature is again the first line of the second transition, which now appears at $336.6 \mathrm{~nm}$. Hence ES(b) is the excitation spectrum of a different molecular species, called species B in the following. When the sample is excited at $336.6 \mathrm{~nm} F S(d)$ is obtained. It consists of two bands, a red band which is similar to that in FS(c), and a blue band which is mirror-symmetric to ES(b). The relative integrated quanturn yields normalized to the red fluorescence band in FS(c) are: $\Phi_{\text {blue }}(\mathrm{c})=1.2 \%, \quad \Phi_{\text {red }}(\mathrm{c})=100 \%, \quad \Phi_{\text {blue }}(\mathrm{d})=$ $3.9 \%$, and $\Phi_{\text {red }}(\mathrm{d})=6.5 \%$.

Since both ES(a) and ES(b) have nonvanishing intensity for both wavelengths 331.9 and $336.6 \mathrm{~nm}$, FS (c) and FS(d) contain contributions from both species. Conversely, if any of the two species should show true dual fluorescence, it should yield a contribution to both excitation spectra. However, we can deduce that species A does not contribute to $\mathrm{ES}(\mathrm{b})$ : The ratio of $\Phi_{\text {blue }}$ (c) to $\Phi_{\text {blue }}$ (d) is $\approx 0.3$. If the blue emission in FS(c) originates from spccics $A$, the linc at $331.9 \mathrm{~nm}$ should appear in ES(b) with an intensity 0.3 times that of the line at $336.6 \mathrm{~nm}$. Since ES (b) shows no trace of such a line, the blue fluorescence in FS(c) must be mainly due to molecules of species B. On the other hand, the sharp line at $336.6 \mathrm{~nm}$ in ES(b) does not appear in ES(a) indicating that the latter is the pure excitation spectrum of species A. This does not rule out the possibility that species $B$ exhibits dual fluorescence, since the concentration of species B (normalized to equal fluorescence quantum yields for both species) is much smaller than that of species A.

The contribution of both species to the fluorescence spectra can be disentangled when the relative intensities of the cxcitation spectra at wavelengths 331.9 and $336.6 \mathrm{~nm}$ are considered. This is indicated in ES(e) for species $A$ and in ES( $f$ ) for species $B{ }^{\# 1}$. The intensity of ES(e) of species A drops by a factor of 0.067 in going from 331.9 to $336.6 \mathrm{~nm}$.

\# Spectra of $\mathrm{c}-\mathrm{f}$ have all been obtained with the same sample and hence refer to a fixed concentration ratio of both species. This ratio could be different for different samples. ES(a) and ES(b) have been obtained with matrices of higher concentration and thickness. As a consequence, the intensity around $331.9 \mathrm{~nm}$ in $\mathrm{ES}$ (b) is too low, since the light of the excitation source is absorbed to a non-negligible extent by the sharp absorption line of species $A$ present in this sample in a large cxcess. 

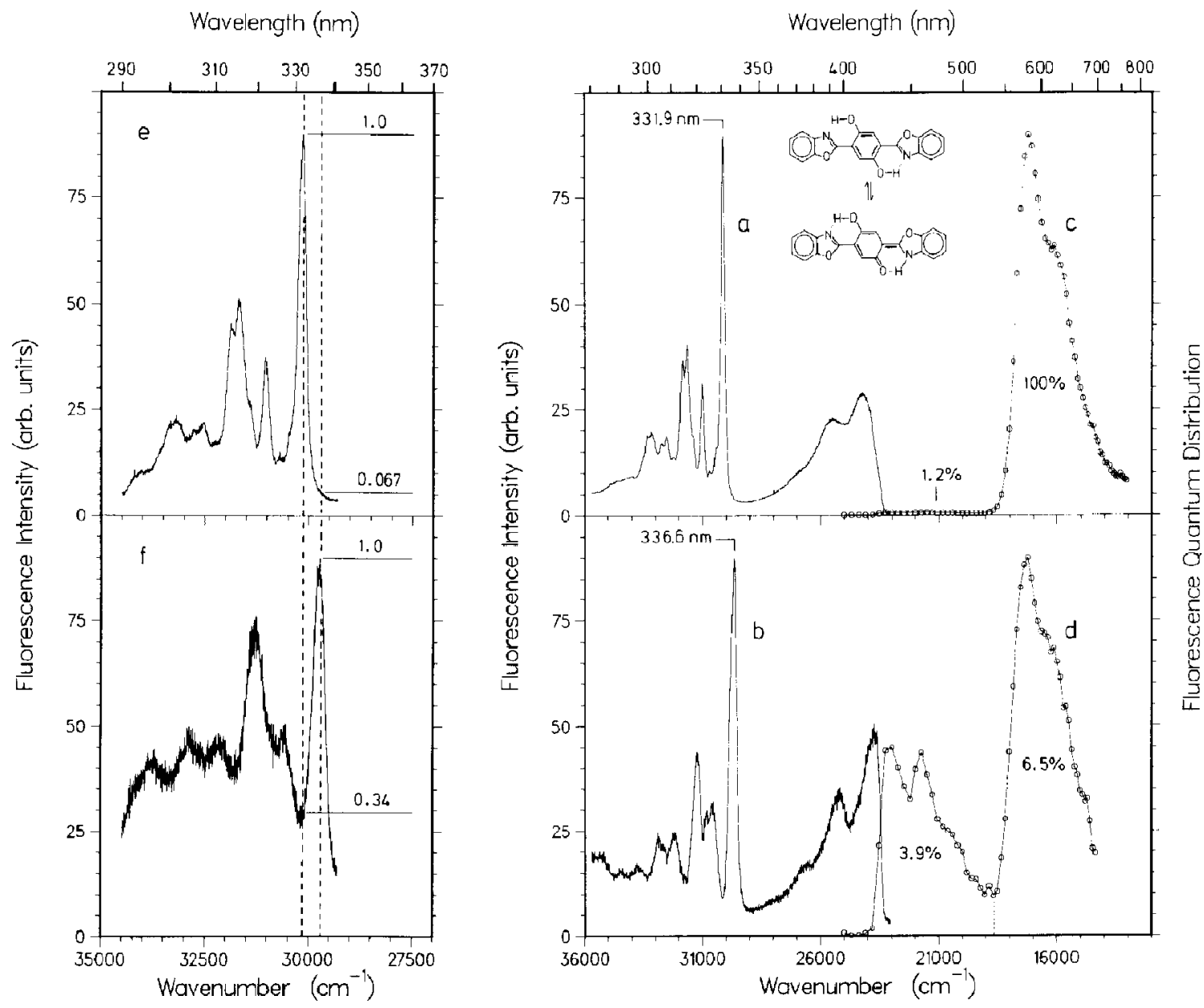

Fig. 1. Spectra of 2,5-bis (2-benzoxazolyl)hydroquinone (BBXHQ) isolated in solid argon. (a, b) Fluorescence excitation spectra detected at 585 and $460 \mathrm{~nm}$, respectively. (c) Quantum distribution of fluorescence (emitted quanta per wavenumber interval) excited into the maximum of the excitation spectrum (a) at $331.9 \mathrm{~nm}$. (d) Quantum distribution of fluorescence excited into the maximum of the excitation spectrum (b) at $336.6 \mathrm{~nm}$. Excitation bandpass for both fluorescence spectra was $0.5 \mathrm{~nm}$, corresponding to the thickness of the dotted vertical lines shown in the left part of the figurc. (c, $f$ ) Expanded fluorescence excitation spectra in the region of the second electronic transition, detected at 585 and $435 \mathrm{~nm}$, respectively. All spectra are corrected for the spectral response of the detection system and the spectrum of the Xe lamp. Spectra $c-f$ were obtained with the same sample.

Hence the yield of red fluorescence should drop to $6.7 \%$, in very good agreement with the observed value of $6.5 \%$. Conversely, the intensity of ES(f) for species B is lower by a factor of 0.34 at $331.9 \mathrm{~nm}$ compared to its value at $336.6 \mathrm{~nm}$. Hence species B should yield a total of $0.34 \times 3.9 \%=1.3 \%$ of blue fluorescence to FS(c), also in very good agreement with the observed value of $1.2 \%$. It is concluded that species $A$ produces only red fluorescence with an estimated ratio $\Phi_{Y} / \Phi_{X}>500$, whereas species $B$ pro- duces only blue fluorescence. The relative amount of species B is $3.9 \%$ when equal fluorescence quantum yields for both species are assumed.

\section{Assignment of the spectra}

GMTY propose the following interpretation of these matrix spectra [6]:

(i) The molecules of species A are 1:1 complexes 
of a BBXHQ molecule with an argon atom.

(ii) The molecules of species $B$ are isolated, doubly fluorescing BBXHQ molecules.

The following arguments are given for these assignments:

(a) Isolatcd BBXHQ molecules in a supersonic jet exhibit dual fluorescence, whereas complexes of BBXHQ with one or more argon atoms show only red fluorescence. This argument is put forward by GMTY without reference, but since they did not publish any jet spectra of BBXHQ they seem to refer to the work of Ernsting [7,8].

(b) The red fluorescence in the FS(d) of fig. 1 is (erroneously) assigned to species B by GMTY. Hence species $B$ is believed to emit dual fluorescence which is also observed with BBXHQ in aprotic solvents at high temperatures.

GMTY believe that the distinction between both species rests in their interaction with argon atoms. The assignment of species B to "isolated" molecules must then imply that these molecules do not interact with even a single argon atom. It is hard to imagine molecules isolated in this strict sense in a solid argon matrix. As has been shown in section 2, the red fluorescence in FS(d) in fig. 1 is entirely due to the large excess of species $A$ in the matrix. This was explicitly stated in ref. [5] and should also be clear from a "careful analysis of the spectra" [6], No ESIPT occurs in molecules of species B. The molecules of species $A$ undergo a very fast ESIPT reaction even at 15 $\mathrm{K}$, apparently without a barrier. Thus none of the two species behaves like BBXHQ at room temperature in aprotic solvents. Two possibilities exist: (1) One of the two species exhibits the typical behavior of isolated BBXHQ at low temperatures and the other species is perturbed. (2) Both species are perturbed.

Several types of perturbation of various strengths could occur:

(a) Probably the weakest interaction that one can think of is solvation of BBXHQ by inert molecules or rare gas atoms. Dipole/induced-dipole interactions should stabilize the tautomer due to its permanent dipole moment and lower any barrier to ESIPT that might exist in the isolated molecule. If the solvent of the absorption spectrum or the dielectric constant $\epsilon$ of the solvent is taken as a measure of solvent-solute interaction, the perturbation should increase in the order $\operatorname{Ar}(\epsilon=1.5)<$ alkanes $(\epsilon=1.9)<$ PMMA $(\epsilon=3.2)<$ methyltetrahydrofuran $(\epsilon=6.2)$. If BBXHQ dissolved in $n$-heptane is regarded as unperturbed, no reason exists to assume a higher degree of perturbation for BBXHQ dissolved in argon.

(b) The occurrence of fast ESIPT depends on the existence of an intramolecular hydrogen bond. Polar impurities can form complexes with an intermolecular hydrogen bond in which the fast ESIPT reaction is inhibited [14]. Formation of such complexes at low temperatures can be avoided through the use of extremely dry solvents [14]. Alternatively, diffusion of the impurities (mostly water) can be inhibited. Thus 3-hydroxyflavone in an argon matrix $[12,13]$ or in PMMA [15] displays strong tautomer fluorescence at $15 \mathrm{~K}$. BBXHQ seems to be less sensitive to this kind of perturbation: The methyltetrahydrofuran which was used as solvent in ref. [4] was not so extensively dried as would have been necessary for 3-hydroxyflavone. Nevertheless, tautomer fluorescence is the dominant emission of $\mathrm{BBXHQ}$ in this

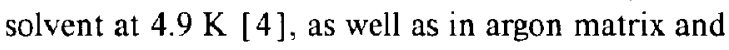
PMMA [15] at $15 \mathrm{~K}$.

(c) Many ESIPT molecules have conformers in which ESIPT is not possible [16,17]. In BBXHQ the benzoxazolyl groups could be rotated by $180^{\circ}$ so that the hydrogen bonds are formed with the oxygen atoms instead of the nitrogen atoms. These conformers have higher energies and hence lower concentration, but could be trapped in low temperature matrices. A conformer with a ground-state enthalpy of $2.7 \mathrm{kcal} / \mathrm{mol}$ above the normal form will exist in $\approx 4 \%$ abundance in the vapor phase at $150^{\circ} \mathrm{C}$ (the condition of matrix deposition for BBXHQ).

\subsection{Assignment of species $A$}

If species $A$ is perturbed this perturbation must enhance ESIPT. This could be due to unspecific solvation by a solvent much more polar than argon, e.g. impurity water molecules. Assuming the wors 1 case that a single water molecule within the first coordination sphere of the BBXHQ molecule is sufficient for this perturbation, the number of unperturbed sites is $P_{0}=(1-p)^{N}$, where $p$ is the mole fraction of water in the matrix and $N$ is the number of next neighbors to the BBXHQ molecule. For $3.9 \%$ of unperturbed sites $\left(P_{0}=0.039\right)$ and an estimated number of $N=40$ 
a mole fraction $p=0.08$ is found, i.e. the matrix must contain $8 \%$ of water. It is probable that the argon matrices contained traces of water, but certainly much less than $8 \%$. So far we are not aware of any kind of perturbation that enhances ESIPT and would account for only $4 \%$ of unperturbed sites. Hence species $A$ is assigned to the unperturbed BBXHQ molecule.

\subsection{Assignment of species $B$}

Consequently, species B must consist of perturbed molecules incapable of ESIPT. Several possible causes exist:

(1) The internal hydrogen bond required for fast ESIPT is broken, either due to complex formation with a hydrogen-bonding impurity, or due to formation of the conformer with the rotated benzoxazolyl group. BBXHQ has two centers capable of ESIPT, and both have to be blocked to account for the exclusive blue fluorescence of species $B$. This implies that a third species should exist in which only one hydrogen bond is blocked. These molecules should undergo efficient ESIPT and contribute to the red fluorescence. If the probability of perturbation is equal and independent for both hydrogen bonds in $\mathrm{BBXHQ}$, a relative amount of $31 \%$ of singly perturbed molecules is calculated. This singly perturbed species should have a strong line in the excitation spectrum of tautomer fluorescence between 331.9 and $336.6 \mathrm{~nm}$, but nothing of this kind is observed.

(2) A conformational change or the site-dependent interaction with solvent molecules modulates the energy separation between the two fluorescing states ${ }^{1} \mathrm{X}^{*}$ and ${ }^{1} \mathrm{Y}^{*}$. In liquid solution the conformational changes and the solvent fluctuations are so rapid that equilibrium is established in $S_{0}$ and $S_{1}$. The molecular ensemble behaves like a single species with average state energies. In rigid solution, however, the ensemble is inhomogeneously distributed: A small fraction of molecules with higher ground-state energy has ' $\mathrm{X}^{*}$ below ${ }^{1} \mathrm{Y}^{*}$. In these molecules ESIPT will not occur at very low temperatures, and they will emit only blue fluorescence. A very similar situation occurs for the ESIPT reaction in the triplet state of 2-(2'-hydroxyphenyl)benzoxazole (HBO) [18]. This behavior would then be an intrinsic property of BBXHQ. The rigid solvent serves only to prevent fast equilibration betwcen the various conformers or sites. Hence this behavior should appear not only in solid argon or solid methyltetrahydrofuran, but in solid alkanes and other solid solvents as well.

(3) The singly excited states of a BBXHQ dimer can as a first approximation be represented as linear combinations of the two corresponding locally excited forms: ${ }^{1}(X X)^{*}={ }^{1}\left(X^{*} X\right) \pm{ }^{1}\left(X X^{*}\right)$ for the normal form and ${ }^{1}(X Y)^{*}={ }^{1}\left(X^{*} Y\right) \pm^{1}\left(X Y^{*}\right)$ for the tautomer. These pairs of states are split by an energy determined by the transition dipoles and the relative orientation of the two BBXHQ molecules. This would account for the red-shift of the excitation spectrum of species B. If this splitting brings the lowest state of the normal form below that of the tautomer, ESIPT cannot occur. The dimer hypothesis is supported by the following observation [19]: A saturated solution of BBXHQ in extremely dry isopentane/cyclopentane $\left(1 ; 4, c \approx 8 \times 10^{-6} \mathrm{M}\right)$ shows dual fluorescence at room temperature. On cooling to $190 \mathrm{~K}$ the red fluorescence vanishes. The blue fluorescence increases and undergoes a red-shift, indicating the formation of a new species. This effect is strongly suppressed when smaller concentrations are used.

\section{On the rate constant for ESIPT in BBXHQ}

\subsection{Results from fluorescence quantum yields}

The schematic diagram representing the reversible ESIPT reaction is

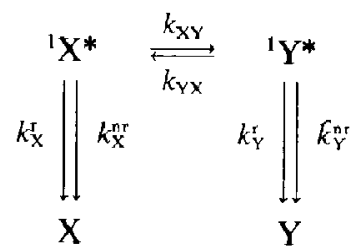

The rate constants for forward and backward ESIPT are $k_{\mathrm{XY}}$ and $k_{\mathrm{YX}}$, those for radiative and nonradiative decay $k^{\mathrm{r}}$ and $k^{\mathrm{nr}}$. Solution of the kinetic equations for this system [20,21] gives the following ratio for the two fluorescence quantum yields:

$\frac{\Phi_{\mathrm{Y}}}{\Phi_{\mathrm{X}}}=\frac{k_{\mathrm{Y}}^{\mathrm{r}} k_{\mathrm{XY}}}{k_{\mathrm{X}}^{\mathrm{r}}\left(k_{\mathrm{Y}}+k_{\mathrm{YX}}\right)}$,

wherc $k_{\mathrm{Y}}=k_{\mathrm{Y}}^{\mathrm{r}}+k_{\mathrm{Y}}^{\mathrm{nr}}$ is the decay rate constant of ${ }^{\mathrm{I}} \mathrm{Y}^{*}$ 
excepting proton transfer. At high temperatures equilibration is much faster than the decay of ${ }^{1} \mathrm{Y}^{*}$ $\left(k_{\mathrm{Y}} \ll k_{\mathrm{YX}}\right)$ and the ratio $\Phi_{\mathrm{Y}} / \Phi_{\mathrm{X}}$ is proportional to the equilibrium constant $K_{\mathrm{e}}(T)=k_{\mathrm{XY}} / k_{\mathrm{YX}}$. Measurement of the temperature dependence of $\Phi_{\mathrm{Y}} / \Phi_{\mathrm{X}}$ yields the reaction enthalpy if the radiative decay constants are temperature-independent. In ref. [3] a reaction enthalpy was determined from the temperature dependence of $\Phi_{\text {blue }}$ (assigned to $\Phi_{\mathrm{X}}$ ) alone. A plot of $\ln \left(1 / \Phi_{\mathrm{X}}\right)$ versus $1 / T$ yielded a straight line with a slope interpreted as a reaction enthalpy of $\Delta H=-0.5 \mathrm{kcal} / \mathrm{mol}$. This required two assumptions, namely that $k_{X} \ll k_{Y}$ and that $k_{Y}$ is independent of temperature. If $k_{Y}$ is temperature-dependent, the observed slope is the sum of the reaction enthalpy and the activation energy of the thermally activated radiationless decay of ${ }^{1} \mathrm{Y}^{*}$. If the value $\Delta H=-0.5 \mathrm{kcal} / \mathrm{mol}$ is used to calculate the ratio of the radiative rate constants, $k_{\mathrm{X}}^{\mathrm{r}} / k_{\mathrm{Y}}^{\mathrm{r}}$, with eq. (1) from the measured quantum yield ratio, a strong temperature dependence is found in contradiction to the assumption made for the analysis (see table 1). On the other hand, if the data of ref. [3] are used to plot $\ln \left(\Phi_{\mathrm{Y}} / \Phi_{\mathrm{X}}\right)$ versus $1 / T$, a curve is obtained with a slope of $-2.3 \mathrm{kcal} / \mathrm{mol}$ between 293 and $190 \mathrm{~K}$, and a slope of $-1.0 \mathrm{kcal} / \mathrm{mol}$ below $190 \mathrm{~K}$. It must be concluded that either the radiative decay constants are strongly temperature-dependent, or that the description of ESIPT as a simple reversible process in BBXHQ is incomplete even in the "high-temperature" region 143-293 K.

The radiative rate constants $k_{\mathrm{X}}^{\mathrm{r}}$ and $k_{\mathrm{Y}}^{\mathrm{r}}$ can be calculated from the data published in refs. $[1,3]$. In the high-temperature approximation $k_{Y}^{r}$ is given by

Table 1

Ratio of the radiative decay constants $k_{\mathrm{X}} / k_{\mathrm{Y}}^{\mathrm{r}}$ calculated from the ratio of fluorescence quantum yields $\Phi_{X} / \Phi_{Y}$

\begin{tabular}{|c|c|c|c|c|c|}
\hline Solvent & $T(\mathrm{~K})$ & $K_{\mathrm{e}}{ }^{a}$ & $\Phi_{X} / \Phi_{Y}$ & $k_{\mathrm{X}}^{\mathrm{1}} / k_{\mathrm{Y}}^{\mathrm{r}}$ & Ref. \\
\hline$n$-heptane & 293 & 2.36 & $0.13^{b)}$ & 0.31 & [6] \\
\hline$n$-heptane & 293 & 2.36 & 0.22 & 0.52 & [1] \\
\hline$n$-heptane & 143 & 5.82 & 0.014 & 0.08 & [1] \\
\hline 3-MP ${ }^{c k}$ & 262 & 2.62 & $0.13^{d y}$ & 0.34 & [3] \\
\hline 3-MP ${ }^{\prime \prime}$ & 138 & 6.21 & $0.008^{4}$ & 0.05 & [3] \\
\hline
\end{tabular}

a) $K_{\mathrm{c}}=\exp (\Delta H / k T), \Delta H=-0.5 \mathrm{kcal} / \mathrm{mol}$.

b) Intcgration of the spectrum (fig. 1 of ref. [6]).

c) 3-methylpentane.

d) Evaluated from data in fig. 1 of ref. [3].
$k_{\mathrm{Y}}^{\mathrm{r}}=\frac{\Phi_{\mathrm{Y}}}{\tau_{\mathrm{X}, \mathrm{Y}}} \frac{1+K_{\mathrm{e}}}{K_{\mathrm{e}}}$,

where $\tau_{X . Y}$ is the common decay time constant of ${ }^{-1} X^{*}$ and ${ }^{1} Y^{*}$. With the experimental data obtained at 143 $\mathrm{K}, \tau_{\mathrm{X}, \mathrm{Y}}=6.2 \mathrm{~ns}, \Phi_{\mathrm{Y}}=0.29$, and $K_{\mathrm{e}}=5.8$, the radiative decay constant $k_{\mathrm{Y}}^{\mathrm{r}}=5.5 \times 10^{7} \mathrm{~s}^{-1}$. This value does not critically depend on the value of $\Delta H$ as long as $K_{\mathrm{e}} \gg 1$. The radiative decay constant $k_{\mathrm{X}}^{\mathrm{r}}$ can be estimated with Förster's formula [22] from the absorption spcctrum in ref. [1] which gives $k_{X}^{r}=1.8 \times 10^{8} \mathrm{~s}^{-1}$. The ratio $k_{X}^{r} / k_{Y}^{r}=3.3$ obtained in this way is quite different from those collected in table 1 which are based on $\Delta H=-0.5 \mathrm{kcal} / \mathrm{mol}$.

At low temperatures $(R T \ll \mid \Delta I I)$ the back-reaction should be frozen in and $k_{\mathrm{YX}}$ can be neglected against $k_{\mathrm{Y}}$ in eq. (1). An estimate of the upper limit $k_{Y X}<10^{13} \mathrm{~s}^{-1} \exp (\Delta H / R T)$ indicates that this is certainly valid below $20 \mathrm{~K}$. Then eq. (1) can be solved for the rate constant of ESIPT, $k_{\mathrm{XY}}$. Unfor" tunately the ratio $k_{X}^{\mathrm{r}} / k_{Y}^{\mathrm{r}}$ is rather uncertain as shown above. With $k_{\mathrm{X}}^{\mathrm{r}} / k_{\mathrm{Y}}^{\mathrm{r}} \approx 1$ and $k_{\mathrm{Y}}=1 / \tau_{\mathrm{Y}}=1.6 \times 10^{8} \mathrm{~s}^{-1}$ [4], for the species $A$ in an argon matrix at $15 \mathrm{~K}$ $\left(\Phi_{Y} / \Phi_{X}>500\right)$ a value of $k_{X Y} \geqslant 8 \times 10^{10} \mathrm{~s}^{-1}$ is obtained.

\subsection{Time-resolved measurements}

In the recent article by GMTY [6], a fast initial decay of the blue fluorescence and a fast rise of the red fluorescence with the same time constant of $\approx 20$ ps have been reported for BBXHQ. This value can only be considered as an upper limit for the following reasons:

(a) The response time of the apparatus defined as the full width at half maximum measured for the scattered laser light is $50 \mathrm{ps}$. Time constants much shorter than 50 ps obtained by deconvolution can be strongly affected by numcrical artifacts.

(b) The residues for the fit to the decay of blue fluorescence show a significant and systematic deviation from zero in the region \pm 50 ps from the peak of the excitation pulse, i.e. where the fast decaying component is calculated. Such behavior could be an artifact caused by uncertainty in the point of zero time delay. In this case the fluorescence rise time is also overestimated. Simulated fluorescence rise curves with a rise time shorter than the response time 
of the apparatus can be superimposed almost exactly by a small shift of the time axis. Also, diffraction at a grating will produce a skewed wavefront with the effect that an instant rise is stretched to an extent that depends on the number of lines illuminated. This effect is not seen for the apparatus response function is it is recorded with the grating in zeroth order. Therefore, fluorescence risetime measurements should be accompanied by measurements of a fluorescence with a similar spectrum but instantaneous rise.

\section{Is there a barrier to ESIPT in BBXHQ?}

The hypothesis of a barrier to ESIPT in BBXHQ can so far only be substantiated by the fact that the two tautomeric forms are in thermal equilibrium at high temperatures. However, this might be a rash judgment. One may ask the question: Is it conceivable that two states of a molecule can be in thermal equilibrium without a separating barrier? Such a situation occurs in many dyes and aromatic ketones with a small energy gap between $T_{1}$ and $S_{1}$, e.g. in the molecule eosin. Following excitation to $S_{1}$ cosin emits phosphorescence and delayed fluorescence decaying with the same time constant [23], indicating that $S_{1}$ and $T_{1}$ are in thermal equilibrium. The triplet yield is not temperature-dependent and intersystem crossing cannot be frozen in. Since the familiar picture of a reaction coordinate cannot be applied to intersystem crossing, it seems that it is not necessary to postulate an energy barrier to account for the coexistcnce of $S_{1}$ and $T_{1}$. With BBXHQ the situation is very similar: ' $\mathrm{X}^{*}$ and ' $\mathrm{Y}$ * coexist at high temperatures, but the reaction cannot be frozen in even at very low temperatures.

One explanation would be to assume an energy barrier which is so low that it can be overcome by proton tunneling with a rate constant of the order of $8 \times 10^{10} \mathrm{~s}^{-1}$. Such a fast tunneling process is indeed conceivable as the following estimate will show. In the model of an inverse parabolic barrier [24], the proton tunnels a length $L$ with a probability

$$
\begin{aligned}
& G=[1+\exp (B)]^{-1}, \\
& B=2 \pi \sqrt{m}\left(V_{0}-W\right) / h \sqrt{A} .
\end{aligned}
$$

In these expressions, $V_{0}$ is the barrier height, $m$ is the mass of the particle, $W$ its energy, and $A=8 V_{0} /$ $L^{2}$. In the molecule HBO, ESIPT occurs in the triplet state as a thermally activated process with a barrier height of $V_{0} \approx 10 \mathrm{kcal} / \mathrm{mol}$. At low temperatures proton tunneling occurs with rate constant $k_{\mathrm{PT}}\left({ }^{3} \mathrm{HBO}\right) \approx 10^{6} \mathrm{~s}^{-1}[25]$. From the observed deuterium isotope effect $G(\mathrm{H}) / G(\mathrm{D})=300$ a value of $B=13.8$ is obtained, leading to estimates $L=0.6 \AA$ and $G_{11 B O}(H)=10^{-6}$. Since the geometries of the hydrogen bonds are very similar in $\mathrm{HBO}$ and BBXHQ, this value of $L$ can be used to estimate $G_{\mathrm{BBXHQ}}(\mathrm{H})=1.2 \times 10^{-2}$ for a barrier height of $V_{0}=1$ $\mathrm{kcal} / \mathrm{mol}$. Assuming equal frequency factors for $\mathrm{HBO}$ and $\mathrm{BBXHQ}$ this yields the estimate $k_{\mathrm{PT}}\left({ }^{1} \mathrm{BBXHQ}\right)=1.2 \times 10^{10} \mathrm{~s}^{-1}$.

Another explanation would be to regard ESIPT not as a reaction along a one-dimensional reaction coordinate, but as a kind of radiationless transition [26].

\section{Conclusion}

(1) Isolation in rare gas matrices produces pure samples for the study of ESIPT reactions in which interactions with the solvent are minimized.

(2) So far no true dual fluorescence has been observed for BBXHQ at low temperatures $(<15 \mathrm{~K}$ ) in any environment except for the free molecule in the supersonic jet.

(3) There is no barrier to ESIPT in BBXHQ, or it is so low that it can be overcome by proton tunneling with a rate constant $k_{X Y} \geqslant 8 \times 10^{10} \mathrm{~s}^{-1}$. Timcresolved measurements of the rise of the tautomer fluorescence or the tautomer transient absorption are necessary to give a definite answer.

\section{Acknowledgement}

The author is indebted to Professor F.P. Schäfer for support of this work. Discussions with Professor W. Lüttke and Dr. N. Ernsting, Dr. K.H. Grellmann, and Dr. B. Nickel are gratefully acknowledged. This work has been supported by the Deutsche For- 
schungsgemeinschaft through the Leibniz-Prize program.

\section{References}

[1]A. Mordzinski, A. Grabowska, W. Kühnle and A. Krowczynski, Chem. Phys. Letters 101 (1983) 291.

[2] A. Mordzinski and A. Grabowska, J. Mol. Struct. 114 (1984) 337.

[3] A. Mordzinski, A. Grabowska and K. Teuchner, Chem. Phys. Letters 111 (1984) 383.

[4] U. Brackman, N.P. Ernsting, D. Ouw and K. Schmitt, Chem. Phys. Letters 110 (1984) 319.

[5] B. Dick, Ber. Bunsenges. Physik. Chem. 91 (1987) 1205.

[6] A. Grabowska, A. Mordzinski, N. Tamai and K. Yoshihara, Chem. Phys. Letters 153 (1988) 389.

[7] N.P. Ernsting, J. Am. Chem. Soc. 107 (1985) 4564.

[8] N.P. Ernsting, J. Phys. Chem. 89 (1985) 4932.

[9] R. Rossetti, R.C. Haddon and L.E. Brus, J. Am. Chem. Soc. 102 (1980) 6913;

R. Rossetti, R. Rayford, R.C. Haddon and L.E. Brus, J. Am. Chem. Soc. 103 (1981) 1303.

[10] V.E. Bondybey, R.C. Haddon and J.H. English, J. Chem. Phys. 80 (1984) 5432;

V.E. Bondybey, R.C. Haddon and P.M. Rentzepis, J. Am. Chem. Soc. 106 (1984) 5969.
[11] Y. Tomioka, M. Ito and N. Mikami, J. Phys. Chem. 87 (1983) 4401.

[12] B. Dick and N.P. Ernsting, J. Phys. Chem. 91 (1987) 4261.

[13] G.A. Brucker and D.F. Kelley, J. Phys. Chem. 91 ( 1987 ) 2856.

[14] D. McMorrow and M. Kasha, J. Phys. Chem. 88 (1984) 2235.

[15] B. Dick, unpublished results.

[16] K. Sandros, Acta Chem. Scand. A 30 (1976) 761.

[17] K.K. Smith and K.J. Kaufmann, J. Phys. Chem. 82 ( (1978) 2286.

[18] B. Nickel and M.F. Rodriguez Prieto, Chem. Phys. Letters 146 (1988) 393.

[19] K.H. Grellmann, unpublished results.

[20] A. Weller, Z. Physik. Chem. NF 15 (1958) 438.

[21] J.B. Birks, Nouv. J. Chim. 1 (1977) 453.

[22] Th. Förster, Fluoreszenz organischer Verbindungen (Vandenhoeck und Ruprecht, Göttingen, 1951) p. 158.

[23] C.A. Parker and C.G. Hatchard, Trans. Faraday Soc. 57 (1961) 1894.

[24] R.P. Bell, The tunnel effect in chemistry (Chapman and Hall, New York, 1980).

[25] K.H. Grellmann, A. Mordzinski and A. Heinrich, to be published.

[26] W. Siebrand, T.A. Wildmann and M.Z. Zgierski, J. Am. Chem. Soc. 106 (1984) 4083, 4089. 